\title{
BAG2 overexpression correlates with growth and poor prognosis of esophageal squamous cell carcinoma
}

https://doi.org/10.1515/biol-2018-0069

Received June 24, 2018; accepted September 29, 2018

\begin{abstract}
Previous studies have suggested that Bcl2associated athanogene 2 (BAG2) serves as a crucial regulator for tumorigenesis in multiple tumors. However, little is known about the effect of BAG2 on esophageal squamous cell carcinoma (ESCC). This study focused on investigating whether BAG2 functions as a cancer-promoting gene in ESCC. In this work, gene expression data and clinical information from the NCBI Gene Expression Omnibus (GEO), Oncomine and The Cancer Genome Atlas (TCGA) were collected and analyzed. Expression of BAG2 in ESCC was determined using quantitative reverse transcription polymerase chain reaction (qRT-PCR). BAG2 was knocked down using small interference RNA (si-RNA) approach. Cell proliferation, migration and invasion were assessed by Cell Counting Kit-8 (CCK-8) and transwell assays. Molecular mechanism was detected by western blotting assay. The expression of BAG2 both in ESCC tissues and cells was upregulated and overexpression was associated with worsened prognosis. BAG2 silencing inhibited ESCC cell proliferation, migration and invasion, which was regulated by the phosphatidylinositol-3-kinase (PI3K)/ protein kinase B (AKT) signaling pathway. These results reveal contributions of BAG2 as a predictor and potential therapeutic target in ESCC.
\end{abstract}

Keywords: Esophageal squamous cell carcinoma, BAG2, Proliferation, Migration, Invasion, Overall survival

\footnotetext{
*Corresponding author: Zheng-Lei Xu, Department of Gastroenterology, Shenzhen People's Hospital, 2nd Clinical Medical College of Jinan University, Shenzhen, Guangdong 518020, P.R.China, E-mail: sumsintry0755@163.com

Ying-Cai Hong, Zheng Wang, Bin Peng, Department of Thoracic Surgery, Shenzhen People's Hospital, 2nd Clinical Medical College of Jinan University, Shenzhen, Guangdong 518020, P.R.China Li-Gang Xia, Lie-Wen Lin, Department of Gastrointestinal Surgery, Shenzhen People's Hospital, 2nd Clinical Medical College of Jinan University, Shenzhen, Guangdong 518020, P.R.Chin
}

\section{Introduction}

With 400,000 estimated deaths each year, esophageal cancer has become the commonest type of malignancy in the digestive system worldwide [1]. In China, esophageal cancer has been ranked as the sixth primary cause of new cancer and the fourth leading cause of cancerrelated deaths [2]. Esophageal squamous cell carcinoma (ESCC) is identified as the most common histologic type of esophageal cancer globally. Despite considerable improvement in cancer therapy regimens over recent decades, the five-year survival rate of ESCC remains unsatisfactory [3], which is largely attributed to a paucity of molecular pathogenesis. Therefore, elucidation of the mechanism underlying the progression of ESCC and the identification of specific biomarkers predicting the prognosis are significant matters regarding the effective strategies and improvements of the treatment of ESCC.

Bcl-2-associated athanogene (BAG)-family proteins, are an evolutionarily conserved family of proteins, originally determined in the study of the anti-apoptosis protein Bcl-2 and participate in multiple functions, for example stress response [4], neuron differentiation [5], cell survival [6] and programmed cell death [7]. BAG2 is identified as a co-chaperone protein and regulates the ubiquitination and degradation of some proteins [8]. It is strongly correlated with neurologic disease, such as Parkinson's disease [9, 10] and Alzheimer's disease [11]. Notably, BAG2 is frequently upregulated in tumors and its overexpression is associated with poor prognosis in patients, including colon cancer, lung carcinoma [12], osteosarcoma [13], fibrosarcoma [14]. BAG2 is also reported to be involved in the pathogenesis of glioblastomas [15]. BAG2 has been shown to participate in pro- oncogenic mechanisms and its overexpression is strongly correlated with progression and poor clinical outcomes of triple-negative breast cancer [16]. It is documented that BAG2 as a micro (mi-) RNA target gene mediates neck squamous cell carcinoma growth and 
apoptosis [17]. Nevertheless, whether and how BAG2 modulates ESCC remained unclear until now.

Hence, the objective of our study was to investigate the expression of BAG2 and the potential role of BAG2 in ESCC progression. In this study, we identified the expression pattern of BAG2 in ESCC tissues or cells by analyzing the data from Gene Expression Ominibus (GEO) and Oncomine datasets. In addition, we determined the association with BAG2 expression and prognosis of ESCC patients. Moreover, the expression of BAG2 in ESCC cells was confirmed. Furthermore, functional experiments were applied to assess the effect of BAG2 on ESCC proliferation, migration and invasion. Molecularly, the underlying mechanism involved in BAG2 influencing ESCC was explored. Taken together, our results may contribute to a theoretical basis for ESCC diagnosis and therapy in the further clinics.

\section{Materials and methods}

\subsection{Data acquisition}

GEO and Oncomine database were used to obtain and analyze BAG2 gene expression data in ESCC and normal samples. The Cancer Genome Atlas (TCGA) dataset was downloaded to analyze the association of BAG2 expression and prognosis. The patients were divided into high expression and low expression groups.

\subsection{Cell culture}

Human ESCC cell lines ECA109, OE19 and normal human esophageal epithelial cell line HEEC were purchased from JENNIO Biological Technology Co. ltd (Guangzhou, China). All cells were cultured in RPMI1640 medium (Thermo Fisher Scientific, Waltham, MA, USA) and was supplemented with $10 \%$ FBS and antibiotics $(100 \mu \mathrm{g} / \mathrm{ml}$ streptomycin and $100 \mathrm{U} / \mathrm{ml}$ penicillin $\mathrm{G}$, Thermo) in a humidified incubator with $5 \% \mathrm{CO}_{2}$ at $37^{\circ} \mathrm{C}$.

\subsection{Cell transfection}

Small interference RNA (siRNA) targeting BAG2 (siBAG2\#1: 5'- CUGCU GUUGA GCAAG AGAA -3'; si-BAG2\#2: 5'- AUCCU UCUGG AAAUG AUCC -3') and its scramble control (si-con) were synthesized by GenePharma Co. ltd (Suzhou, China). Cell transfection was performed using Lipofectamine 2000 reagent (Thermo) according to the protocol of manufacturer. The knockdown of the targeting gene was confirmed by both quantitative reverse transcription polymerase chain reaction (qRTPCR) and western blotting analyses.

\subsection{Total RNA extraction and qRT-PCR}

Total RNA was extracted from cells using TRIZOL reagent (Thermo) following the manufacturer's instructions. RNAs were reverse-transcribed to single-strand cDNAs using PrimeScript ${ }^{\mathrm{TM}} \mathrm{RT}$ reagent kit and then amplified using SYBR Premix Ex Taq kit (TaKaRa Bio, Shiga, Japan) on an ABI-7900 Real-Time PCR Detection System (7900HT, Applied Biosystems, Foster city, CA, USA) in accordance with manufacturer's protocols. GAPDH was used as an endogenous control. The primer sequences used were as follows: BAG2: F: 5'- CTAGC CACAT TAGGC GCTCG -3', R: 5'- GCGGC TGGAG TTAGA GGTTC -3'; GAPDH: F: 5'- GGAGC GAGAT CCCTC CAAA AT -3'; R: 5'- GGCTGTTGTCATACTTCTCATGG -3'. The relative expression level of BAG2 was calculated using the $2^{-\Delta \Delta C t}$ method. Experiments were carried out in triplicate.

\subsection{Cell Counting Kit-8 (CCK-8) assay}

ECA109 cells $\left(1 \times 10^{4}\right)$ were seeded into 96-well plates overnight. At $0,24,48,72$ or 96 h post-transfection, $10 \mu$ Cell Counting Kit-8 solution (Dojindo Molecular Technologies, Kumamoto, Japan) was added into each well, followed by additional incubation of $1 \mathrm{~h}$ at $37^{\circ} \mathrm{C}$. The absorbance was measured by optical density at the wavelength of $450 \mathrm{~nm}$ (OD450) to assess cell proliferation capability.

\subsection{Transwell migration and invasion assay}

Transwell assay was performed to measure the migration and invasion of ECA109 cells using transwell chambers with polycarbonate membrane (Corning, NY, USA). For invasion assay, the membrane was coated with Matrigel (BD Bioscience, San Jose, CA, USA). The $200 \mu \mathrm{L}$ cell suspension containing $1 \times 10^{4}$ cells in FBS-free medium was plated in the upper chamber and $600 \mu \mathrm{L}$ medium containing 10\% FBS was added in the lower chamber. After $24 \mathrm{~h}$ of culture, the membrane was washed with PBS, fixed with $4 \%$ paraformaldehyde, and stained with $0.1 \%$ crystal violet. After washing with water, the cell numbers on the bottom sides of the membrane 
were counted in five randomly selected fields under an inverted microscope at $200 \times$ magnification. The migration assay was performed as previous described only without Matrigel. The experiments were carried out in triplicate.

\subsection{Western blotting analysis}

Cellswereextracted from usingradioimmunoprecipitation (RIPA) lysis buffer (Beyotime) and then quantified using a BCA Protein Assay Kit (Beyotime). Proteins $(20 \mu \mathrm{g})$ were separated by $10 \%$ sodium dodecyl sulphate-polyacrylamide gel electrophoresis (SDSPAGE) and transferred to polyvinylidene fluoride (PVDF) membranes for $1.5 \mathrm{~h}$ at $4^{\circ} \mathrm{C}$. Then, membranes were blocked with $5 \%$ skim milk in TBST at room temperature for $1.5 \mathrm{~h}$. The membranes were incubated with primary antibodies against BAG2 (1:1000, Thermo), phosphatidylinositol-3-kinase (PI3K), phosphorylated(p-)PI3K, protein kinase B (AKT), p-AKT and GAPDH (1:1000, Cell Signaling Technology, Boston, MA, USA). GAPDH was used as the loading control. The blots were developed with an enhanced chemiluminescence reagent (Thermo) and exposed on a ChemiDoc MP imager (Bio-Rad Laboratories). Quantity One software (Bio-Rad Laboratories) was used to quantify the band density. The experiments were performed three times.

\subsection{Statistics}

Survival curves were plotted using the Kaplan-Meier method, and the differences were analyzed by the logrank statistic. Student's t-test was used to compare the difference of data in different groups. $P$-values of less than 0.05 presented the difference was statistically significant. All statistical analyses were performed using SPSS (version 22.0, SPSS Inc., Chicago, IL, USA) and GraphPad Prism 6.0 (GraphPad Software Inc, LaJolla, CA, USA). The results are representative of three independent experiments.

Ethical approval: The conducted research is not related to either human or animals use.

\section{Results}

\subsection{BAG2 expression is upregulated in human ESCC tissue samples and cell lines}

By comparing the data related with expression of BAG2 in ESCC tissues and normal samples obtained from GEO and Oncomine dataset, mRNA level of BAG2 was highly expressed in ESCC tissues as compared with normal samples (Fig. 1A-C, $P<0.01$ ). To further investigate the biological function of BAG2, we assessed the expression of BAG2 in ESCC cell lines, including ECA109 and OE19 and normal human esophageal epithelial cell line HEEC. Consistently, the mRNA expression level of BAG2 in these ESCC cell lines was significantly upregulate on comparing HEEC (Fig. 1D, $P<0.01$ ). ECA109 was chosen to further investigate the role of BAG2, based on the result the mRNA expression level of BAG2 is the highest amongst these ESCC cell lines. These data imply that BAG2 is of importance in ESCC progression.

\subsection{BAG2 high expression is correlated with poor survival}

By analyzing the data obtained from the TCGA dataset, the overall survival (OS) rate of the ESCC patients with high BAG2 expression was significantly poorer than that of the patients with low BAG2 expression $(P=0.038$; Fig. 2$)$.

\subsection{Knockdown of BAG2 in ESCC cells inhibits cell proliferation}

To determine the effect to biological behavior of overexpression ofBAG2inESCCcells, weused siRNA to knock down BAG2 expression in ECA109 cells. The suppression of BAG2 by siRNAs was checked using qRT-PCR and western blotting analyses. In ECA109 cells which overexpress BAG2, the expression level of the BAG2 mRNA and protein was more efficiently diminished after the transfection with the si-BAG2\#1/2 than with the si-con (Fig. $3 \mathrm{~A}$ and B, $P<0.01$ ). Based on the repressive effect of si-BAG2\#1 on ECA109 cells, we used it to perform subsequent experiments. In order to assess the oncogenic role of BAG2, we first conducted a CCK-8 cell proliferation assay. The OD values in ESCC cells were markedly reduced in si-BAG2 group compared with si-con group after transfection of 48, $72 \mathrm{~h}$ and $96 \mathrm{~h}$ (Fig. 3C, $P<0.01)$. Overall, these results suggest that BAG2 regulates the proliferation of ESCC. 
A

$\mathrm{P}=0.003$

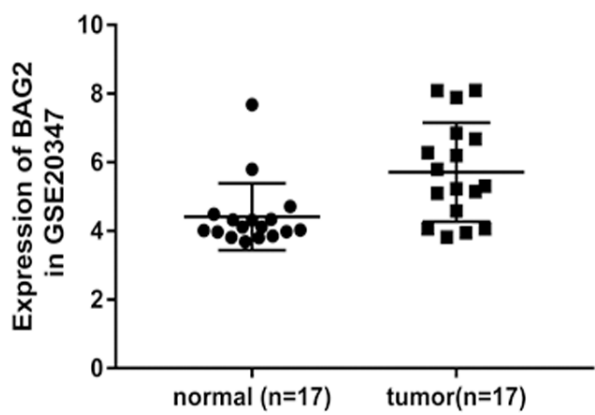

C

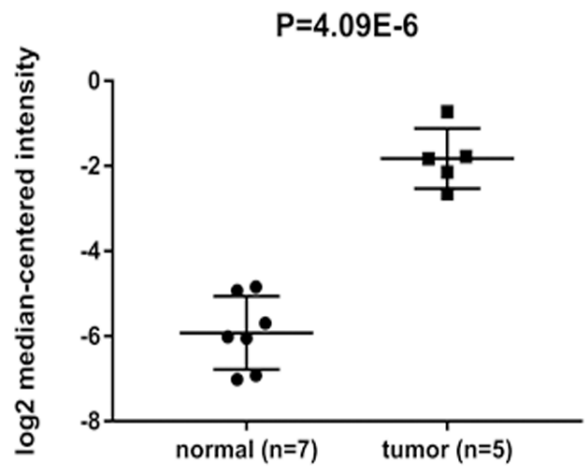

B

$P=0.000725$

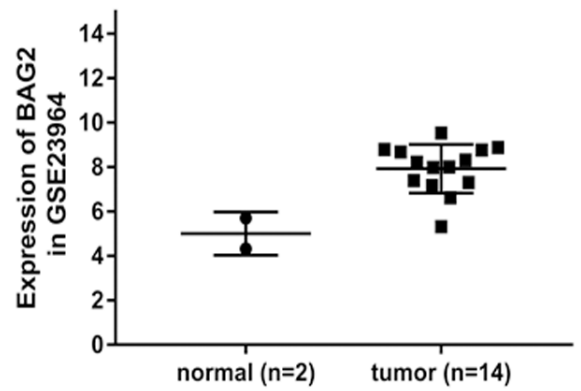

D

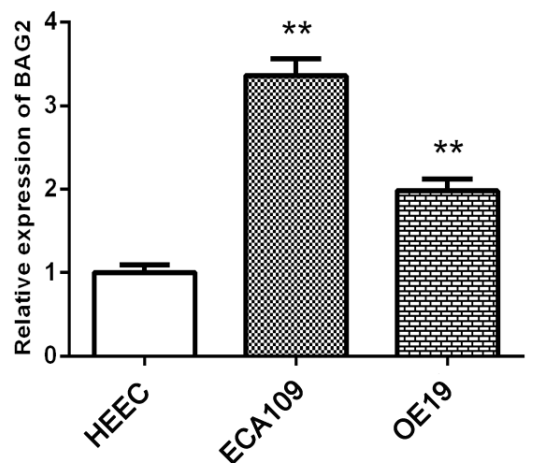

Figure 1. Significantly upregulated expression of BAG2 in ESCC tissues and cells. A-C, BAG2 was highly overexpressed in ESCC tissues or cells compared with normal samples based on the data from GEO and Oncomine datasets. D, BAG2 was detectably elevated in ESCC cells in contrast with normal cells. Results are expressed as the mean \pm SD of three independent experiments. ${ }^{\star *}: P<0.01$.

\subsection{Depletion of BAG2 expression represses ESCC cells migration and invasion}

Next, to further test whether BAG2 expression has an effect on ESCC cell migration and invasion, we investigated cellular migration and invasion by transwell assays. Transwell migration and invasion assays showed that the number of migrated or invaded cell was dramatically decreased in si-BAG2 group compared with si-con group (Fig. 4, $P<0.01$ ). These results indicate that BAG2 silencing is capable of suppressing both migration and invasion of ESCC cells.

\subsection{Suppression of PI3K/AKT signaling pathway by the knockdown of BAG2}

In an effort to gain insight into the mechanistic basis of BAG2 in ESCC, we assessed the effect of BAG2 silencing on activity of PI3K/AKT signaling, on the basis that PI3K/AKT

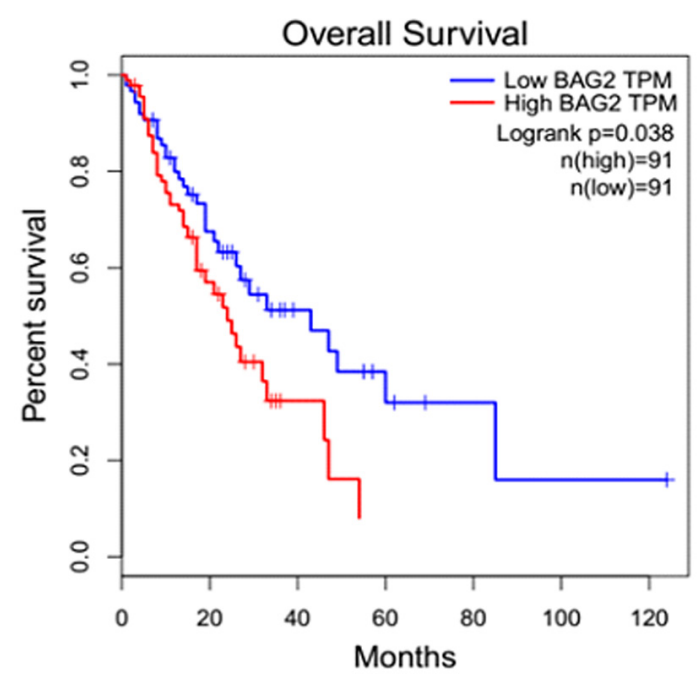

Figure 2. Overall survival (OS) of ESCC patients according to BAG2 expression. The OS rates of the ESCC patients in the high BAG2 expression group was markedly lower than that in the patients with low BAG2 expression. 
A

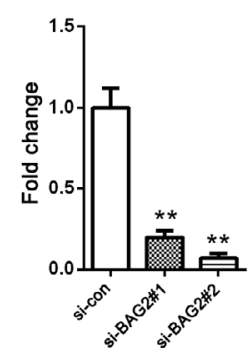

B

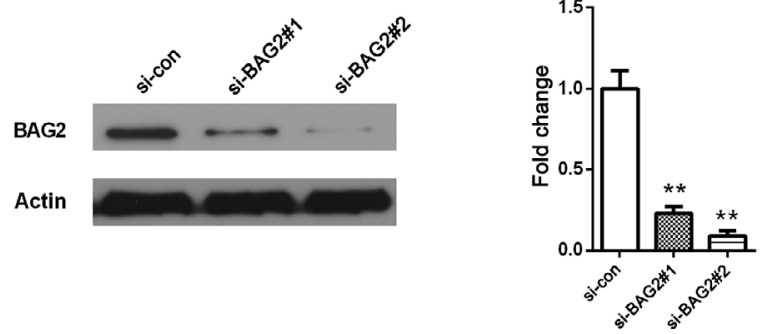

C

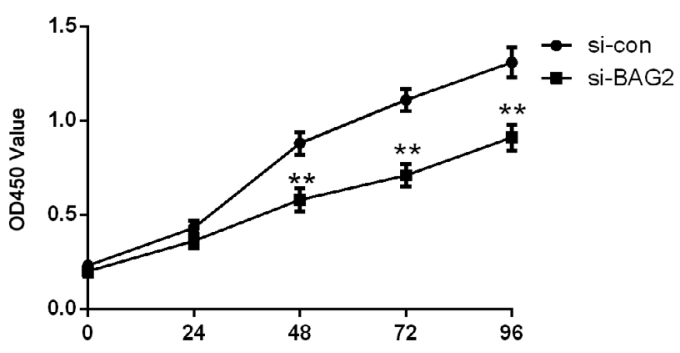

Figure 3. Proliferation of ESCC cells was inhibited by BAG2 silencing. A-B, mRNA and protein level of BAG2 was lower in ESCC cells treated with si-BAG2\#1/2 than that in si-con. C, The OD values were decreased in ESCC cells transfected with si-BAG2. Results are expressed as the mean \pm SD of three independent experiments. ${ }^{\star *}: P<0.01$.

signaling is of significance in various tumors initiation and progression. A significant reduction in p-PI3K and p-AKT, not PI3K and AKT, was demonstrated in ESCC cells that were treated with si-BAG2 compared with those transfected with si-con (Fig. 5, P $<0.01$ ). Altogether, these data indicate that BAG2 plays an oncogenic role in ESCC by affecting cell proliferation, migration and invasion, which is modulated by PI3K/AKT signaling pathway.

\section{Discussion}

In the current work, our investigations herein demonstrated that BAG2 was highly overexpressed both in ESCC tissues and cells. Our data also showed that overexpression of BAG2 correlated with poorer OS of ESCC patients. Additionally, knockdown of BAG2 expression using si-RNA approach exhibited a repressive effect of ESCC cell proliferation, migration and invasion, which was possibly regulated by suppression of PI3K/AKT signaling. These observations imply that BAG2 may function as a potential target for treating or preventing ESCC.

Mounting evidence has confirmed that carcinogenesis is a complicated process involving large numbers of molecules and biological pathways important for cancer development. In this study, upregulated BAG2 in ESCC tissues and cells was identified through bioinformatics

A

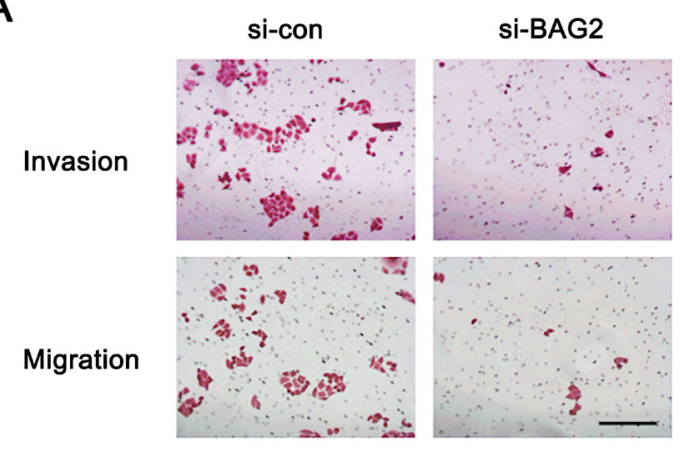

B

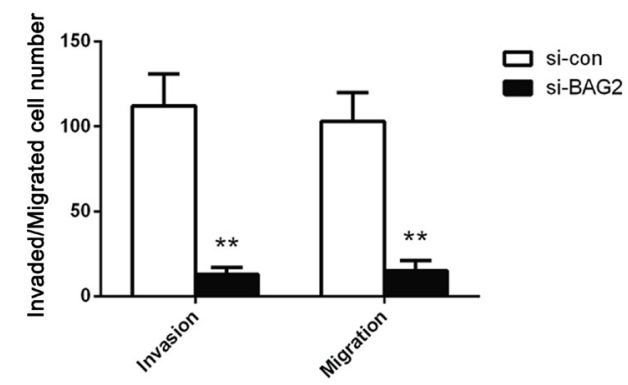

Figure 4. Knockdown of BAG2 repressed the migration and invasion of ESCC cells. A, Transwell migration and invasion assays were applied to evaluate the motility of ESCC cells. B, The migrated and invaded cell number of ESCC cells was quantified. Original magnifications: $\times 200$. Results are expressed as the mean \pm SD of three 
independent experiments. ${ }^{*}: P<0.01$.

A

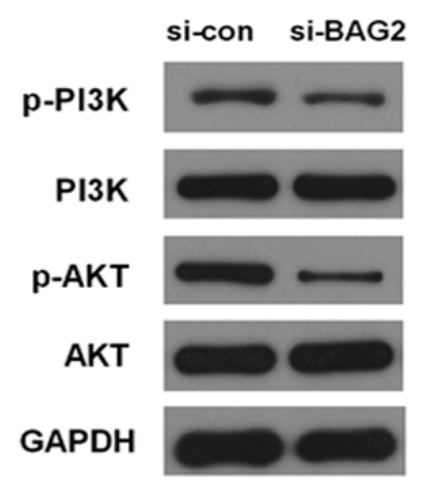

B

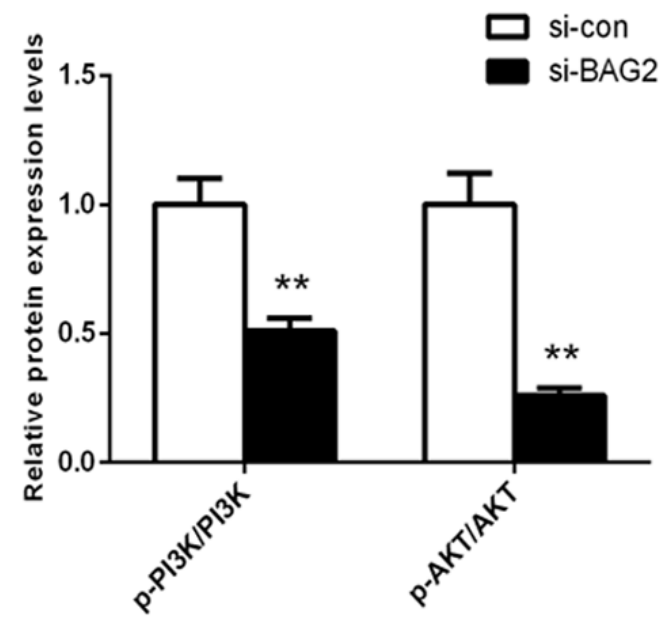

Figure 5. Knockdown of BAG2 suppressed the activation of PI3K/AKT pathway in ESCC cells. A, Total proteins were subjected to western blot analysis using PI3K, p-PI3K, AKT and p-AKT antibodies. B, Quantification analysis was performed using Quantity One software.

and qRT-PCR analyses. Significantly, overexpression of BAG2 was closely associated with poorer prognosis of ESCC patients as compared with samples of low-expression of BAG2. These data indicate a pro-oncogenic role of BAG2 in ESCC. Of note, current studies have demonstrated that BAG2 is highly expressed in various types of cancers, namely, osteosarcoma, fibrosarcoma, et al., in addition, high-level of BAG2 is correlated with poor outcome of clinic tumor patients $[14,18]$. Collectively, previous studies in combination with our current work suggest a potential oncogenic role of BAG2 in ESCC.

Studies in some of cancers have suggested that BAG2 plays a vital role in tumorigenesis via involving in tumor growth [17, 19]. Kyung-Min et al have documented that BAG2 expression induces metastasis of triple-negative breast cancer [16]. It is well-established that unbridled proliferation and metastasis are the characteristics of tumorigenesis [20, 21]. Hereby, to further verify the oncogenic role of BAG2 in ESCC, we carried out wound-healing and transwell assays to determine ESCC proliferation, migration and invasion after treatment with si-BAG2. Results showed that markedly decreased proliferation, migration and invasion of ESCC was found in si-BAG2 group as compared with si-con group. This evidence indicates that BAG2 might act as a significant oncogene in ESCC development.

Numerous studies have revealed that PI3K/AKT signaling pathway is responsible for occurrence and development of tumors [22, 23]. Previous research has confirmed that activation of the PI3K/AKT pathway contributes to the progression of ESCC, including facilitating cell viability, abilities of colony formation and cell migration [24]. Moreover, PI3K, p-PI3K, AKT and p-AKT are significantly upregulated in tumor tissues. Additionally, some of these molecules are correlated with OS and progression-free survival and even are proved to be an independent adverse prognostic factor for OS in ESCC [25]. Huang et al have reported that dual mTORC1 and MTORC2 inhibitor could exhibit effective antitumor action of ESCC [26]. To gain insight of the underlying mechanism of BAG2 regulation of ESCC progression, we assessed the expression of critical oncogenic members of the signaling pathways including PI3K, p-PI3K, AKT and p-AKT. The results obtained from western blotting analysis showed that p-PI3K and p-AKT, not PI3K and AKT, were obviously decreased in si-BAG2 group on comparing si-con group. Overall, these data also suggest a likely mechanism by which BAG2 exerts a pro-oncogenic action of ESCC. However, a limitation of the study is that only one cell line was used to perform most of functional experiments. There is also a lack of animal evidence. Thus, additional research is required. Although there are several limitations to this study, our preliminary data offer a theoretical basis for ESCC pathogenesis and aid to unearth a novel therapeutic management for ESCC.

In conclusion, this is the first report suggesting that BAG2 plays a vital role in ESCC progression. Our results showed that elevated expression of BAG2 was identified in ESCC tissues and cells, in addition, overexpression of BAG2 was closely associated with worsened OS of ESCC patients. Functional analyses suggested that BAG2 downregulation could inhibit ESCC cell proliferation, migration and invasion, which was linked with PI3K/ AKT signaling repression. Our findings provide evidence 
that BAG2 might serve as an important oncogene in ESCC progression, thus targeting it predicts a promising therapeutic strategy for ESCC patients.

Conflict of interest: Authors state no conflict of interest.

\section{References}

[1] Torre LA, Bray F, Siegel RL, Ferlay J, Lortet-Tieulent J, Jemal A. Global cancer statistics, 2012. CA: a cancer journal for clinicians. 2015;65:87-108.

[2] Chen W, Zheng R, Baade PD, Zhang S, Zeng H, Bray F, et al. Cancer statistics in China, 2015. CA: a cancer journal for clinicians. 2016;66:115-132.

[3] Castro C, Bosetti C, Malvezzi M, Bertuccio P, Levi F, Negri E, et al. Patterns and trends in esophageal cancer mortality and incidence in Europe (1980-2011) and predictions to 2015. Annals of oncology : official journal of the European Society for Medical Oncology. 2014;25:283-290.

[4] Piltti J, Bygdell J, Qu C, Lammi MJ. Effects of long-term low oxygen tension in human chondrosarcoma cells. Journal of cellular biochemistry. 2018;119:2320-2332.

[5] Patil SP, Tran N, Geekiyanage H, Liu L, Chan C. Curcumininduced upregulation of the anti-tau cochaperone BAG2 in primary rat cortical neurons. Neuroscience letters. 2013;554:121-125.

[6] Li S, Banck M, Mujtaba S, Zhou MM, Sugrue MM, Walsh MJ. p53-induced growth arrest is regulated by the mitochondrial SirT3 deacetylase. PLoS One. 2010;5:e10486.

[7] Fang S, Li L, Cui B, Men S, Shen Y, Yang X. Structural insight into plant programmed cell death mediated by BAG proteins in Arabidopsis thaliana. Acta crystallographica. Section D, Biological crystallography. 2013;69:934-945.

[8] Arndt V, Daniel C, Nastainczyk W, Alberti S, Hohfeld J. BAG-2 acts as an inhibitor of the chaperone-associated ubiquitin ligase CHIP. Molecular biology of the cell. 2005;16:5891-5900.

[9] Che X, Tang B, Wang X, Chen D, Yan X, Jiang H, et al. The BAG2 protein stabilises PINK1 by decreasing its ubiquitination. Biochemical and biophysical research communications. 2013;441:488-492.

[10] Qu D, Hage A, Don-Carolis K, Huang E, Joselin A, Safarpour F, et al. BAG2 Gene-mediated Regulation of PINK1 Protein Is Critical for Mitochondrial Translocation of PARKIN and Neuronal Survival. The Journal of biological chemistry. 2015;290:3044130452.

[11] Carrettiero DC, Hernandez I, Neveu P, Papagiannakopoulos T, Kosik KS. The cochaperone BAG2 sweeps paired helical filament- insoluble tau from the microtubule. The Journal of neuroscience : the official journal of the Society for Neuroscience. 2009;29:2151-2161.

[12] Yue X, Zhao Y, Liu J, Zhang C, Yu H, Wang J, et al. BAG2 promotes tumorigenesis through enhancing mutant $\mathrm{p} 53$ protein levels and function. eLife. 2015;4: doi:10.7554.

[13] Jian Y, Chen Y, Geng C, Liu N, Yang G, Liu J, et al. Target and resistance-related proteins of recombinant mutant human tumor necrosis factor-related apoptosis-inducing ligand on myeloma cell lines. Biomedical reports. 2016;4:723-727.
[14] Klopfleisch R, Meyer A, Lenze D, Hummel M, Gruber AD. Canine cutaneous peripheral nerve sheath tumours versus fibrosarcomas can be differentiated by neuroectodermal marker genes in their transcriptome. Journal of comparative pathology. 2013;148:197-205.

[15] Asperger A, Renner C, Menzel M, Gebhardt R, Meixensberger J, Gaunitz F. Identification of factors involved in the anti-tumor activity of carnosine on glioblastomas using a proteomics approach. Cancer investigation. 2011;29:272-281.

[16] Yang KM, Bae E, Ahn SG, Pang K, Park Y, Park J, et al. Co-chaperone BAG2 Determines the Pro-oncogenic Role of Cathepsin B in Triple-Negative Breast Cancer Cells. Cell reports. 2017;21:2952-2964.

[17] Ray RB, Hauser B, Zhao Y, Pang X, Ling Z, Myers E, et al. Functions of MiRNA-128 on the Regulation of Head and Neck Squamous Cell Carcinoma Growth and Apoptosis. Plos One. 2015;10:e0116321.

[18] Andersen MH, Becker JC, Thor Straten P. The antiapoptotic member of the Bcl-2 family Mcl-1 is a CTL target in cancer patients. Leukemia. 2005;19:484-485.

[19] Zhu D, Gao W, Zhang Z. MicroRNA-1180 is associated with growth and apoptosis in prostate cancer via TNF receptor associated factor 1 expression regulation and nuclear factorkappaB signaling pathway activation. Oncology letters. 2018;15:4775-4780.

[20] Bao Y, Zhang S, Guo Y, Wei X, Zhang Y, Yang Y, et al. Stromal expression of JNK1 and VDR is associated with the prognosis of esophageal squamous cell carcinoma. 2018

[21] Jin H, Qiao F, Chen L, Lu C, Xu L, Gao X. Serum metabolomic signatures of lymph node metastasis of esophageal squamous cell carcinoma. Journal of proteome research. 2014;13:4091-4103.

[22] Xi R, Pan S, Chen X, Hui B, Zhang L, Fu S, et al. HPV16 E6-E7 induces cancer stem-like cells phenotypes in esophageal squamous cell carcinoma through the activation of PI3K/ Akt signaling pathway in vitro and in vivo. Oncotarget. 2016;7:57050-57065.

[23] Chen J, Lan T, Zhang W, Dong L, Kang N, Fu M, et al. Dasatinib enhances cisplatin sensitivity in human esophageal squamous cell carcinoma (ESCC) cells via suppression of PI3K/AKT and Stat 3 pathways. Archives of biochemistry and biophysics. 2015;575:38-45.

[24] Liu M, Hu Y, Zhang MF, Luo KJ, Xie XY, Wen J, et al. MMP1 promotes tumor growth and metastasis in esophageal squamous cell carcinoma. Cancer letters. 2016;377:97-104.

[25] Wu N, Du Z, Zhu Y, Song Y, Pang L, Chen Z. The Expression and Prognostic Impact of the PI3K/AKT/mTOR Signaling Pathway in Advanced Esophageal Squamous Cell Carcinoma. 2018;17:1533033818758772.

[26] Huang Y, Xi Q, Chen Y, Wang J, Peng P, Xia S, et al. A dual mTORC1 and mTORC2 inhibitor shows antitumor activity in esophageal squamous cell carcinoma cells and sensitizes them to cisplatin. Anti-cancer drugs. 2013;24:889-898. 